\title{
Cibermedios y web 2.0: modelo de análisis y resultados de aplicación
}

\author{
Por Ruth Rodríguez-Martínez, Lluís Codina y Rafael Pedraza-Jiménez
}

\begin{abstract}
Resumen: Se propone un modelo de análisis para cibermedios (periodismo digital) con el fin de determinar su calidad en relación con un conjunto de parámetros generales y, en especial, respecto a la adopción de la web 2.0. Este modelo se ha testado con el análisis de ocho diarios digitales cuyos resultados se presentan también. Presenta un carácter modular que permite por un lado su extensión, y por otro, su aplicación total o parcial en contextos de análisis diferentes.
\end{abstract}

Palabras clave: Prensa digital, Ciberperiodismo, Noticias online, Medios de comunicación, Web 2.0, Web social, Adopción de la web 2.0.

Title: Online journalism and web 2.0: A model for analysis and report on results

Abstract: A model is proposed for analyzing online journalism from the perspective of suitability for certain quality parameters

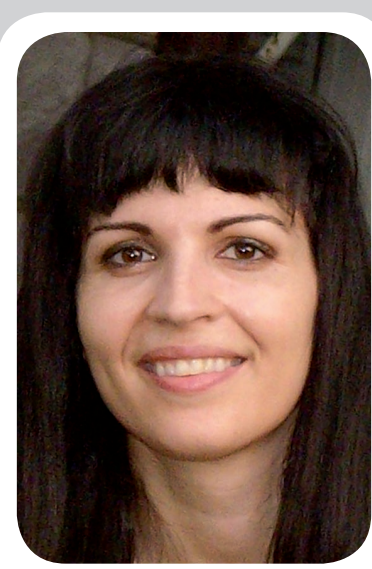

Ruth Rodríguez-Martínez es doctora en periodismo por la Universidad Complutense de Madrid. Sus principales líneas de investigación se enmarcan en el periodismo cultural y el periodismo online. Imparte docencia en los Estudios de Periodismo y en el Interdisciplinary Master in Cognitive Systems and Interactive Media de la Universitat Pompeu Fabra de Barcelona. Ha realizado una estancia de investigación postdoctoral en la School of Journalism de la University of Missouri (EUA) y participa en diversos proyectos de investigación con financiación pública.

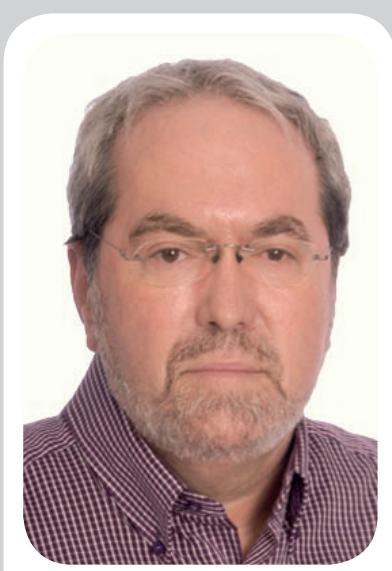

Lluís Codina es profesor titular del Departamento de Comunicación de la Universitat Pompeu Fabra (UPF) y director de la Unidad de Soporte a la Calidad y a la Innovación Docente (Usquid) de la Facultad de Comunicación de la UPF. Imparte docencia en las titulaciones de Periodismo y de Comunicación Audiovisual. Es coordinador del Grupo de Investigación DigiDoc de la UPF y codirector del Máster Online en Buscadores y del Máster Online en Documentación Digital. Participa en el Máster Interuniversitario UB-UPF sobre Gestión de Contenidos Digitales.

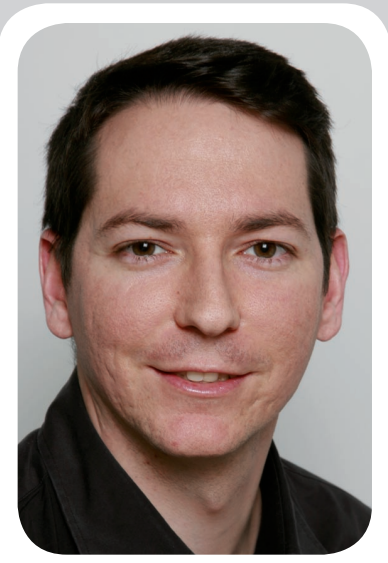

Rafael Pedraza-Jiménez es profesor de la UPF y miembro del grupo de investigación DigiDoc de esta misma universidad. Imparte docencia en los estudios de Periodismo y Comunicación Audiovisual, así como en el master interuniversitario en Gestión de Contenidos Digitales (UB-UPF), el master en Buscadores (IDEC) UPF) y el master online en Documentación Digital (IDEC/ UPF). Participa en diversos proyectos de investigación, y sus principales líneas de interés incluyen la web semántica, ingeniería de ontologías, recuperación de información, y técnicas de SEO/SEM.

and, especially, in relation to certain indicators on the adoption of the characteristic features of web 2.0. This model has been tested with the analysis of eight online newspapers and the results are presented. The model has a modular nature, which permits its application, in whole or in part, to other research projects.

Keywords: Online journalism, Online news, Online newspapers, Mass communication, Web 2.0, Social web, Web 2.0 adoption.

Rodríguez-Martínez, Ruth; Codina, Lluís; Pedraza-Jiménez, Rafael. “Cibermedios y web 2.0: modelo de análisis y resultados de aplicación”. El profesional de la información, 2010, enero-febrero, v. 19, n. 1, pp. 35-44.

DOI: 10.3145/epi.2010.ene.05

\section{Introducción}

El objetivo de este trabajo es proponer un modelo de análisis de los cibermedios, prestando especial atención a los diarios digitales. Es evidente que se pueden establecer conjuntos exhaustivos de indicadores sin ningún límite previo; sin embargo, es útil disponer de un modelo de evaluación relativamente sencillo que permita análisis comparativos con un amplio número de medios. Para refinar y testar esta propuesta, ha sido aplicada a ocho diarios ya que uno de los motivos de este trabajo, primero de una serie en el seno de un proyecto de investigación sobre cibermedios, es la presentación del modelo en sí mismo y probar y/o demostrar su posible viabilidad en otros contextos.

Con tal motivo, desarrollando y ampliando una línea anterior de trabajo de algunos de los autores, se 
propone un conjunto de indicadores específicos para valorar determinados aspectos de calidad formal de los diarios digitales, incluyendo un estudio sobre la adopción de la web 2.0 por parte de la prensa digital. Por último se aplica el modelo de análisis a ocho medios: cinco españoles y tres de otros países.

\section{"Entre los distintos contenidos que pueden encontrarse en la Web, las noticias ocupan un lugar cada vez más destacado"}

\section{Cibermedios}

El uso de internet aumenta incesantemente, por lo que cabe preguntarse de qué forma y cómo ha variado su utilización en los últimos años. En este sentido, en 2003 el 46\% de los usuarios de Estados Unidos la concebían como una herramienta de comunicación (por ejemplo, mediante el correo electrónico), mientras que el $34 \%$ de los encuestados la utilizaban para buscar contenidos. En cambio, en 2008 los resultados fueron bien distintos: el $28 \%$ la utilizaba la como un medio para comunicarse, pero el número de internautas que afirmaba usar internet para acceder a contenidos había ascendido al $45 \%$ (datos publicados por la Online $\mathrm{Pu}$ blishers Association).

\section{http://www.online-publishers.org/}

Entre los contenidos que pueden encontrarse en la Web las noticias ocupan un lugar cada vez más destacado. Según el estudio elaborado por el Pew Internet \& American Life Project, en agosto de 2008 cuatro de cada diez estadounidenses encuestados utilizaba la Red para informarse. Estos datos llevaron al Pew Research Center a afirmar que la Red se ha convertido en el soporte con mayor crecimiento de audiencia, ya que el resto de formatos ve cómo ésta se mantiene o incluso desciende.

Este aumento de consumo de noticias en la Red parece deberse en gran medida al ejercicio de adaptación al formato web que han llevado a cabo los medios de comunicación durante los últimos años. Periódicos, revistas y canales de televisión han cambiado notablemente su actitud respecto a la Web. Los sitios web de los medios han aumentado progresivamente la cantidad de contenidos, al tiempo que los han hecho más atractivos. Y aunque fieles a la imagen del periódico o del canal de televisión en cuestión, sus versiones en línea han creado un tipo de estilo y lenguaje propios pensados para satisfacer las de- mandas de los nuevos usuarios en línea (Horrigan, 2006). Esto explica que el consumo de televisión haya aumentado, ya que al acceso tradicional se ha sumado el acceso desde la Red, o que un gran número de internautas haya dejado de pasar las páginas de los periódicos manualmente para hacerlo a golpe de clic. Centrándonos en el caso de la prensa digital, diferentes estudios demuestran que los lectores en línea valoran especialmente:

- facilidad de acceso a la noticia: pueden encontrar fácilmente lo que buscan y en el momento que lo desean.

- personalización de los contenidos o informaciones recibidas: sobre todo a partir de servicios de alerta o de canales de contenido sindicado (rss, atom, etc.).

- constante actualización de la información: prácticamente al minuto, de manera que pueden conocerse en todo momento las noticias de última hora.

- confianza: es un factor en el que la Web ha ganado en los últimos años (según datos publicados en 2006 por Pew Research Center, el 55\% de los internautas encuestados consideraba la información que encontraban en la Red correcta y fiable; esta cifra no ha descendido).

\section{"Los sitios web de los medios han aumentado progresivamente la cantidad de contenidos, al tiempo que los han hecho más atractivos"}

A estos factores habría que añadir la gratuidad (en muchos casos) del acceso a las noticias.

Afortunadamente contamos ya con una cierta cantidad de aportaciones teóricas y de trabajos sobre aspectos diversos de los cibermedios. Algunos de los realizados en Estados Unidos ya se han mencionado, y se pueden añadir los que viene realizando desde hace tres años The Bivings Group (ver bibliografía). En el caso de España merece la pena destacar los análisis realizados por los profesores Javier Díaz-Noci (actualmente en la Universitat Pompeu Fabra) y Ramón Salaverría (Universidad de Navarra), aunque no son los únicos ni mucho menos, como es fácil comprobar por la bibliografía de este artículo, que en absoluto pretende ser exhaustiva. Por último cabe mencionar el amplio trabajo publicado en 2005 consistente en una comparativa de la prensa impresa y en línea de 16 países europeos, entre ellos España (Wurff; Lauf; O'Sullivan, 2005). 


\section{Calidad de los contenidos en línea de la prensa digital}

De todo lo dicho podemos concluir que los lectores de diarios han encontrado en la Web un entorno propicio para el acceso a la noticia. Ahora bien, ¿hasta qué punto los diarios digitales han sabido adaptarse a los cambios recientes de este escenario, en particular a la aparición de la serie de elementos que configuran la web 2.0?

Con el fin de determinarlo, en primer lugar se propondrá un modelo de análisis mediante un conjunto de indicadores $\mathrm{y}$, seguidamente, se ha seleccionado una muestra de ocho sitios web de algunos de los principales diarios de España y del mundo a los que se ha aplicado este modelo. Los análisis se repitieron en días alternos (de lunes a viernes) durante el mes de noviembre de 2009. Dado que en este trabajo no se analizan contenidos sino características del medio digital, la repetición se hizo únicamente para asegurar la consistencia de los datos.

\section{"Los diarios han ido aumentando progresivamente su presencia en la Web, hasta el punto de que hoy es difícil encontrar un diario que no posea una versión en línea"}

El criterio de selección ha sido el siguiente: se han seleccionado los sitios web de cinco diarios españoles de información general de referencia, tanto en formato papel como digital. A fin de disponer de un elemento de comparación, se les ha añadido un diario de referencia de Inglaterra, Francia y Estados Unidos. La muestra de análisis la forman $A b c, E l$ mundo, El país, El periódico, La vanguardia, Le monde, The guardian y The New York times.

\section{http://www.abc.es/ \\ http://www.elmundo.es/ \\ http://www.elpais.com/ \\ http://www.elperiodico.com/ \\ http://www.lavanguardia.es/ \\ http://www.lemonde.fr/ \\ http://www.guardian.co.uk/ \\ http://www.nytimes.com/}

El estudio ha analizado la página principal y todas las secciones de los diarios seleccionados, con el fin de valorar la calidad de los contenidos de sus sitios web. Para la identificación y descripción de los indicadores se ha tomado como punto de partida la propuesta pre- sentada en "Metodología de análisis y evaluación de recursos digitales en línea" (Codina, 2006).

Los indicadores se dividen en dos grupos:

\section{Generales:}

Valoran aspectos que toda web, con independencia de su temática, género, objetivos, etc., debe cuidar.

\section{Especificos:}

- Internos, propios, y a la vez característicos de los sitios web de los medios de comunicación: valoran elementos concretos que el sitio de un diario en línea debiera incluir.

- Externos pero vinculados a los medios y que permiten observar el impacto de la web social en la prensa digital.

Todos los análisis se llevaron a cabo durante las tres primeras semanas de noviembre de 2009, siendo comprobados los datos de cada tabla en diversas ocasiones. Las fechas concretas indicadas en cada tabla $(3,4,17,20$ y 25 de noviembre) corresponden en cada caso a las últimas comprobaciones realizadas. Dado el carácter estructural de los indicadores analizados, que se refieren al medio y no a la noticia, y el carácter independiente de cada uno, entendemos que en este tipo de evaluaciones no es incoherente llevar a cabo los análisis en fechas independientes para cada indicador, siempre que la dispersión de fechas sea muy limitada como es el caso.

\section{Indicadores generales}

Han sido objeto de análisis la accesibilidad del sitio web, su visibilidad y popularidad, la calidad de su código fuente, y los puntos de acceso a la información que presentan. Los resultados se muestran a continuación.

\section{"Se ha analizado la accesibilidad, visibilidad y popularidad, calidad del código fuente, y puntos de acceso a la información de cada web"}

\section{Accesibilidad}

En primer lugar se ha evaluado la accesibilidad (Voces-Merayo, 2007) de los sitios web de los diarios, es decir, el nivel de adecuación de sus contenidos a los usuarios con características especiales, como deficiencias visuales, auditivas, o motrices, o que utilicen tecnologías de capacidad limitada (como agendas electrónicas y teléfonos móviles). Como es sabido, existen 
tres niveles de adecuación: A, doble A (AA), y triple A (AAA).

El nivel de adecuación se determina en función del grado de cumplimento de las reglas elaboradas por la Iniciativa para la Accesibilidad Web (WAI) del World Wide Web Consortium (W3C). De este modo, los requisitos para considerar una web accesible se dividen en: a) requisitos de prioridad 1: aquellos que "tiene" que satisfacer para que los usuarios con necesidades especiales puedan acceder a la información (su consecución implica un nivel de adecuación A); b) requisitos de prioridad 2: aquellos que "debe" cumplir para facilitar su accesibilidad (nivel de adecuación AA); c) requisitos de prioridad 3: aquellos que "puede" cumplir para optimizar la accesibilidad de sus usuarios (nivel de adecuación AAA). Idealmente, para llegar a la mayor cantidad posible de lectores e informarles, un diario debería implementar su web conforme a un nivel de adecuación AAA.

La tabla 1 muestra los resultados. En ella se recoge el número de errores detectados automáticamente por el Test de accesibilidad web (Taw), en cada sitio y para cada nivel de adecuación.

http://www.tawdis.net

Los resultados obtenidos son sorprendentes, ya que sólo uno de los sitios analizados satisface el nivel mínimo de accesibilidad a sus contenidos (el diario británico The guardian). Es más, el elevado número de errores en la codificación hace suponer que, o bien para estos diarios la accesibilidad de sus contenidos no es una prioridad, o desconocen la importancia que tiene la adecuada codificación de sus páginas web para facilitar el acceso universal a sus noticias.

\section{Visibilidad y popularidad}

El segundo aspecto analizado es la visibilidad y la popularidad de la página principal de los diarios. La visibilidad se determina en función del número de enlaces que esta página recibe de otros sitios web (y de la calidad de los mismos). Es un aspecto muy importante ya que nos indica cuán fácil será encontrar este diario en la Red, principalmente mediante el uso de buscadores. La popularidad hace referencia al número de visitas que recibe un sitio y el número de páginas servidas por él. Ambas medidas nos ayudan a determinar la importancia o el impacto de los diarios digitales en el conjunto de la Web.

Para realizar este análisis se han utilizado las siguientes medidas:

- PageRank de Google: valora la visibilidad de un sitio web mediante una puntuación que va de 0 a 10 , siendo 10 la puntuación óptima.

- TrafficRank de Alexa: determina la popularidad de un sitio web, asignándole una posición en el ranking mundial (y nacional) de las webs más visitadas que elabora Alexa. La puntuación óptima sería 1, es decir, ser la página más popular (visitada) del mundo. Una página es más popular cuanto menor es su TrafficRank.

- Número de enlaces que recibe cada sitio web según Yahoo!: cuanto mayor es el número de enlaces que recibe más probable es que aparezca entre los primeros resultados recuperados por un motor de búsqueda. Por tanto, un número elevado es muy positivo.

- Número de páginas web indizadas por Yahoo! de cada sitio: esta medida nos permite conocer cuán-

\section{"Es importante para los medios adaptar sus contenidos a los nuevos formatos y plataformas, para tener mayor difusión de sus noticias y captar nuevas audiencias"}

\begin{tabular}{|l|c|c|c|c|}
\hline \multirow{2}{*}{} & \multicolumn{4}{|c|}{$\begin{array}{c}\text { Número de errores de accesibilidad } \\
\text { (mediante el test Taw) }\end{array}$} \\
\cline { 2 - 5 } & Prioridad 1 & Prioridad 2 & Prioridad 3 & Total \\
\hline http://www.elpais.com/ & 18 & 110 & 9 & 137 \\
\hline http://www.elmundo.es/ & 41 & 179 & 15 & 235 \\
\hline http://www.elperiodico.com/ & 16 & 118 & 6 & 140 \\
\hline http://www.lavanguardia.es/ & 54 & 112 & 6 & 172 \\
\hline http://www.abc.es/ & 53 & 134 & 4 & 191 \\
\hline http://www.lemonde.fr/ & 48 & 120 & 13 & 181 \\
\hline http://www.guardian.co.uk/ & 0 & 9 & 2 & 11 \\
\hline http://www.nytimes.com/ & 28 & 93 & 7 & 128 \\
\hline
\end{tabular}

Tabla 1. Nivel de accesibilidad (página principal) (análisis 03/1 1/2009) tas páginas de un sitio web son visibles a los buscadores (es decir, están indexadas por ellos), y en consecuencia cuántas pueden ser recuperadas por un buscador. De nuevo es muy positivo un elevado número.

Nótese que para la extracción tanto del número de enlaces que reciben los diarios como del número de páginas que tienen indexadas se ha utilizado el índice internacional de Yahoo!, y no de Google. 


\begin{tabular}{|c|c|c|c|c|c|}
\hline \multirow{2}{*}{ Medio } & \multirow{2}{*}{$\begin{array}{c}\text { PageRank } \\
\text { (Google) }\end{array}$} & \multicolumn{2}{|c|}{$\begin{array}{l}\text { TrafficRank } \\
\text { (Alexa) }\end{array}$} & \multirow{2}{*}{$\begin{array}{l}\text { Enlaces } \\
\text { recibidos } \\
\text { (Yahoo!) }\end{array}$} & \multirow{2}{*}{$\begin{array}{c}\text { Páginas } \\
\text { indexadas } \\
\text { (Yahoo!) }\end{array}$} \\
\hline & & $\begin{array}{c}\text { Ranking } \\
\text { internacional }\end{array}$ & $\begin{array}{l}\text { Ranking } \\
\text { nacional }\end{array}$ & & \\
\hline http://www.elpais.com/ & 8 & 624 & 17 & 2.662 .521 & 856.901 \\
\hline http://www.elmundo.es/ & 9 & 456 & 15 & 3.481 .958 & 2.455 .046 \\
\hline http://www.elperiodico.com/ & 4 & 7.243 & 188 & 2.395 .714 & 307.576 \\
\hline http://www.lavanguardia.es/ & 4 & 5.551 & 151 & 1.138 .751 & 747.655 \\
\hline http://www.abc.es/ & 7 & 3.184 & 92 & 2.217 .328 & 549.341 \\
\hline http://www.lemonde.fr/ & 8 & 973 & 60 & 3.124 .739 & 562.207 \\
\hline http://www.guardian.co.uk/ & 9 & 263 & 18 & 3.591 .334 & 1.643 .842 \\
\hline http://www.nytimes.com/ & 9 & 102 & 27 & 11.143 .702 & 3.299 .799 \\
\hline
\end{tabular}

Tabla 2. Visibilidad y popularidad (análisis 04/11/2009)

Esta decisión está motivada por la mayor consistencia de los datos del primero (en este sentido hemos seguido una práctica habitual en este tipo de análisis). La tabla 2 muestra los resultados obtenidos.

El análisis de los resultados revela que la mayoría de estos diarios han cuidado la visibilidad y la popularidad de sus contenidos. Especialmente relevantes son los casos de The New York times (con las mejores puntuaciones) y The guardian, y también de El mundo (con los mejores resultados en el ámbito nacional español) y El país.

El resto de medios españoles obtienen una baja puntuación, siendo especialmente negativos los resultados

\section{"El análisis de los resultados obtenidos revela que la mayoría de estos diarios cuidan la visibilidad y popularidad de sus contenidos"}

web. Concretamente se ha examinado si los diarios digitales disponen de: búsqueda simple, búsqueda avanzada, hemeroteca y mapa del sitio web. Los resultados pueden verse en la tabla 3 .

El resultado es muy positivo ya que en casi todos los sitios analizados aparecen las formas de acceso a la información señaladas. Estos datos podrían ser indicativos de la importancia que tiene para los diarios en línea facilitar el acceso a sus noticias.

\section{Indicadores específicos internos}

Para completar el análisis de estos sitios web es necesario estudiar las particularidades propias de los diarios en línea. Para ello se ha de proponer previamente un conjunto de indicadores específicos que, atendiendo a los contenidos, el público y los objetivos de estos sitios, nos ayuden a identificar cuáles son sus características más deseables.

Este trabajo propone un conjunto de veintitrés indicadores específicos internos, agrupados en tres categorías. Se presentan a modo de pregunta, consideran-

de El periódico y La vanguardia, que contrastan enormemente con la importancia y tirada de sus versiones impresas. Similar aunque algo mejor es la situación de $A b c$. En los tres casos podría ser conveniente la definición de una estrategia de posicionamiento que les ayudara a mejorar su visibilidad en la Web.

\section{Acceso a la información}

El último indicador general estudiado son las formas de acceso al contenido en estos sitios

\begin{tabular}{|l|c|c|c|c|}
\hline \multicolumn{1}{|c|}{ Medio } & $\begin{array}{c}\text { Bús- } \\
\text { queda } \\
\text { simple }\end{array}$ & $\begin{array}{c}\text { Búsque- } \\
\text { da avan- } \\
\text { zada }\end{array}$ & $\begin{array}{c}\text { Hemero- } \\
\text { teca }\end{array}$ & $\begin{array}{c}\text { Mapa de } \\
\text { la web }\end{array}$ \\
\hline http://www.elpais.com/ & Sí & Sí & Sí & No \\
\hline http://www.elmundo.es/ & Sí & Sí & Sí & Sí \\
\hline http://www.elperiodico.com/ & Sí & No & Sí & Sí \\
\hline http://www.lavanguardia.es/ & Sí & Sí & Sí & No \\
\hline http://www.abc.es/ & Sí & Sí & Sí & Sí \\
\hline http://www.lemonde.fr/ & Sí & No & Sí & Sí \\
\hline http://www.guardian.co.uk/ & Sí & Sí & Sí & No \\
\hline http://www.nytimes.com/ & Sí & Sí & Sí & Sí \\
\hline
\end{tabular}

Tabla 3. Puntos de acceso a la información en los diarios digitales (análisis 17/11/2009) 
do su respuesta afirmativa un rasgo positivo del medio analizado, y su respuesta negativa una carencia que debiera ser suplida. Dado que el análisis llevado a cabo ha tenido como objeto de estudio la página principal (home o portada) y las distintas secciones, la ausencia de los indicadores en las portadas no ha derivado en un resultado negativo. De igual modo, la aparición de un indicador una única vez no ha sido suficiente para considerarlo de forma positiva.

Son los siguientes:

\section{Categoría 1: Profundización en la información}

- ¿Aparece la hora de la última actualización? (En tabla: Hora actual) ticia)

- ¿Se identifica al autor de la noticia? (Autor no-

- Cuando se accede al texto de una noticia ¿hay enlaces a artículos de la misma sección? (Enlace sección)

- Cuando se accede al texto de una noticia ¿hay enlaces a artículos de la hemeroteca digital? (Enlace hemer.)

- ¿Se facilitan enlaces a artículos o informaciones externas al propio diario para contextualizar las noticias? (Enlace externo)

- ¿Es posible acceder a los artículos de un autor concreto? (Art. autor)

- ¿Se emplea contenido gráfico para cubrir o ampliar la cobertura de las noticias? (Fotografía/infografía)

- ¿Se emplea contenido en formato de audio o vídeo para cubrir o ampliar la cobertura de las noticias? (Audio/vídeo)

Los resultados revelan que sólo dos diarios satisfacen la totalidad de los indicadores estudiados, The New York times y La vanguardia, aunque es muy positivo comprobar que los demás satisfacen la mayoría de los indicadores.

\section{Categoría 2: Herramientas de interacción}

- ¿Dispone el diario de un blog? (En tabla: Blog)

- ¿Ofrece la posibilidad de crear un blog a sus usuarios dentro de su propio dominio? (Blog usuarios)

- ¿Se pueden hacer comentarios a las noticias publicadas? (Comentarios)

- ¿Dispone de herramientas web 2.0 para compartir contenidos? (Compartir)

- ¿Permite a los lectores contactar vía correo electrónico con el autor de una noticia? (Contactar autor)

- ¿Acepta contribuciones de los lectores? (Contribuciones de los lectores)

- ¿Dispone de foros de discusión? (Foro)

- ¿Tiene chats para sus usuarios? (Chat)

- ¿Posee una sección con las noticias más leídas? $($ Noticias + leídas $)$

El estudio de estos indicadores revela que prácticamente la totalidad de las herramientas de interacción analizadas han sido incorporadas a los sitios web de los diarios digitales. Para la selección de indicadores nos hemos basado en un trabajo anterior de los autores (Rodríguez-Martínez; Pedraza-Jiménez, 2009).

Categoría 3: Personalización de la información.

- ¿Posee un servicio de alertas? (En tabla: Alertas)

- ¿Dispone de canales de contenido sindicado? (Rss)

- ¿Es posible el envío de noticias vía correo electrónico? (e-mail)

- ¿Facilita el envío de noticias a teléfonos móviles? (Móvil)

\begin{tabular}{|c|c|c|c|c|c|c|c|c|}
\hline Medio & $\begin{array}{c}\text { Hora } \\
\text { actual }\end{array}$ & $\begin{array}{l}\text { Autor } \\
\text { noticia }\end{array}$ & $\begin{array}{l}\text { Enlace } \\
\text { sección }\end{array}$ & $\begin{array}{l}\text { Enlace } \\
\text { hemer. }\end{array}$ & $\begin{array}{c}\text { Enlace } \\
\text { externo }\end{array}$ & $\begin{array}{l}\text { Art. } \\
\text { autor }\end{array}$ & $\begin{array}{c}\text { Fotografía/ } \\
\text { infografía }\end{array}$ & $\begin{array}{c}\text { Audio/ } \\
\text { vídeo }\end{array}$ \\
\hline http://www.elpais.com/ & Sí & Sí & Sí & Sí & Sí & No & Sí & Sí \\
\hline http://www.elmundo.es/ & Sí & Sí & No & Sí & No & No & Sí & Sí \\
\hline http://www.elperiodico.com/ & Sí & Sí & Sí & Sí & No & No & Sí & Sí \\
\hline http://www.lavanguardia.es/ & Sí & Sí & Sí & Sí & Sí & Sí & Sí & Sí \\
\hline http://www.abc.es/ & Sí & Sí & Sí & Sí & No & No & Sí & Sí \\
\hline http://www.lemonde.fr/ & Sí & Sí & Sí & Sí & No & No & Sí & Sí \\
\hline http://www.guardian.co.uk/ & Sí & Sí & Sí & Sí & No & Sí & Sí & Sí \\
\hline http://www.nytimes.com/ & Sí & Sí & Sí & Sí & Sí & Sí & Sí & Sí \\
\hline
\end{tabular}

Tabla 4. Profundización en la información (análisis 17/11/2009) 


\begin{tabular}{|c|c|c|c|c|c|c|c|c|c|}
\hline Medio & Blog & $\begin{array}{c}\text { Blog } \\
\text { usuarios }\end{array}$ & $\begin{array}{c}\text { Comen- } \\
\text { tarios }\end{array}$ & $\underset{\text { tir }}{\text { Compar- }}$ & $\begin{array}{c}\text { Contactar } \\
\text { autor }\end{array}$ & $\begin{array}{l}\text { Contri- } \\
\text { buciones } \\
\text { de los } \\
\text { lectores }\end{array}$ & Foro & Chat & $\begin{array}{l}\text { Noticias } \\
\text { + leídas }\end{array}$ \\
\hline http://www.elpais.com/ & Sí & Sí & Sí & Sí & No & Sí & Sí & Sí & Sí \\
\hline http://www.elmundo.es/ & Sí & Sí & Sí & Sí & No & Sí & Sí & Sí & Sí \\
\hline http://www.elperiodico.com/ & Sí & Sí & Sí & Sí & No & Sí & Sí & No & Sí \\
\hline http://www.lavanguardia.es/ & Sí & Sí & Sí & Sí & Sí & Sí & Sí & No & Sí \\
\hline http://www.abc.es/ & Sí & No & Sí & Sí & No & Sí & Sí & Sí & Sí \\
\hline http://www.lemonde.fr/ & Sí & Sí & Sí & Sí & No & Sí & Sí & Sí & Sí \\
\hline http://www.guardian.co.uk/ & Sí & No & Sí & Sí & No & Sí & Sí & Sí & Sí \\
\hline http://www.nytimes.com/ & Sí & No & Sí & Sí & Sí & Sí & Sí & Sí & Sí \\
\hline
\end{tabular}

Tabla 5. Herramientas de interacción (análisis 17/1 1/2009)

- ¿Ofrece a sus usuarios la opción de registrarse? (Registro)

- ¿Cuenta con aplicaciones que permitan a sus usuarios personalizar los contenidos del diario? (Personalizar)

Como se puede apreciar en la tabla 6, la mayor parte de los medios han optado por utilizar canales alternativos a su sitio web para la difusión de sus contenidos, ya sea mediante el correo electrónico o el móvil. De esta manera los periódicos potencian la idea de que el usuario puede acceder a la información siempre que quiera y desde el soporte que más se adecue a sus necesidades.

Por otro lado, todos los diarios analizados ofrecen a sus lectores la opción de recibir alertas sobre aquellos temas o secciones que sean de su interés. Este tipo de feedback que llega de los usuarios, inimaginable en la prensa impresa, hace que los periodistas puedan observar cuáles son las secciones o temas que más aceptación tienen entre los lectores. Gracias a este tipo de servicios los medios pueden analizar además cómo las preferencias de los lectores cambian, ya sea en función de sus motivaciones personales o externas. Así por ejemplo, durante una campaña electoral es probable que la suscripción a alertas de la sección nacional aumente; o el triunfo de un equipo de fútbol incremente el número de lectores interesados en recibir noticias relacionadas.

Otro de los indicadores que ha sido mayoritariamente aceptados por los medios online es el del registro de usuario. Esto presenta una nueva forma de acceder a la información en la medida en que el usuario deja de ser anónimo para el medio.

\section{Indicadores específicos externos}

Hasta hace poco contar con una amplia comunidad de usuarios sólo estaba al alcance de algunos privilegiados, pero la llegada de la web $2.0 \mathrm{y}$, sobre todo, de las tecnologías que facilitan la comunicación social (como foros, blogs, redes sociales, etc.), ha dotado a todos los agentes de la Web de herramientas para:

- Atraer y fidelizar usuarios a un dominio web. Esto se logra mediante la creación de sitios web con contenido fácilmente accesible y atractivo para su audiencia. En este sentido son importantes las herramientas que un diario digital facilita a sus usuarios para que interactúen entre ellos y con los contenidos.

\begin{tabular}{|c|c|c|c|c|c|c|}
\hline Medio & Alertas & Rss & e-mail & Móvil & Registro & Personalizar \\
\hline http://www.elpais.com/ & Sí & Sí & Sí & Sí & Sí & Sí \\
\hline http://www.elmundo.es/ & Sí & Sí & Sí & Sí & Sí & Sí \\
\hline http://www.elperiodico.com/ & Sí & Sí & Sí & Sí & Sí & Sí \\
\hline http://www.lavanguardia.es/ & Sí & Sí & Sí & Sí & Sí & No \\
\hline http://www.abc.es/ & Sí & Sí & Sí & Sí & Sí & Sí \\
\hline http://www.lemonde.fr/ & Sí & Sí & Sí & Sí & Sí & Sí \\
\hline http://www.guardian.co.uk/ & Sí & Sí & Sí & Sí & Sí & Sí \\
\hline http://www.nytimes.com/ & Sí & Sí & Sí & Sí & Sí & Sí \\
\hline
\end{tabular}

Tabla 6. Personalización de la información (análisis 17/11/2009).
- Difundir los contenidos más allá del propio sitio web. Es posible gracias al uso de aplicaciones de comunicación que permiten buscar y acceder a potenciales usuarios de los servicios en línea ofrecidos por el medio. Esto supone un cambio fundamental en el proceso de comunicación web. Ahora el papel de los proveedores de contenidos, los diarios en línea entre otros, 
no sólo consiste en que los usuarios lleguen a su sitio web, sino también en implementar estrategias para que sus contenidos lleguen hasta sus usuarios. De esta manera la noticia se traslada desde un contexto puramente periodístico, como es el diario en línea, a un contexto social donde el medio actúa como un usuario más.

Entre las herramientas que ayudan a la consecución del primer objetivo se encuentran blogs, canales de contenido sindicado, foros, chats, etc. Son herramientas que, como hemos podido comprobar en el análisis anterior, ya facilitan los principales diarios en línea.

Por otro lado, para lograr el segundo objetivo es muy conveniente el uso de servicios tales como:

- Redes sociales

- Servicios para el alojamiento de contenido (multimedia)

- Servicios de microblogging

- etc.

Los indicadores específicos estudiados en el anterior epígrafe tienen como rasgo común que han sido analizados en los sitios web de los medios de comunicación seleccionados. Sin embargo los que se proponen a continuación se caracterizan porque se encuentran vinculados a estos medios pero no aparecen en sus propios sitios web. Para los indicadores de este epígrafe nos hemos basado en un trabajo anterior de los autores (Rodríguez-Martínez; Pedraza-Jiménez, 2009).

Para analizarlos ha sido preciso visitar los sitios web de diversas redes sociales y plataformas de la web

\section{"Se constata una tendencia generalizada a la incorporación de los diarios a plataformas sociales como Facebook, Twitter, Flickr o Youtube"}

A la luz de estos resultados podemos afirmar que en general los diarios analizados están presentes en las redes sociales más populares. Esta presencia muestra la tendencia que tanto medios de comunicación como empresas en general han llevado a la práctica con el fin de captar usuarios y generar una comunidad en torno a ellos.

\section{"El éxito de la prensa digital depende de su capacidad para crear sitios web que permitan la interacción tanto de los usuarios con los contenidos, como de los usuarios entre sí"}

También se ha estudiado el uso que los diarios hacen de las nuevas plataformas web 2.0 y sus formatos de comunicación. La web 2.0 ha propiciado la aparición de multitud de servicios especializados en el alojamiento y la difusión de contenidos con un formato específico. Es destacable el éxito que han alcanzado los servicios de alojamiento de vídeos como Youtube o de imágenes como Flickr. La utilización de este tipo de servicios proporciona a los medios nuevos canales de comunicación que les acercan a sus potenciales usuarios y les facilitan la creación de auténticas comunidades. Un buen ejemplo sería el canal de vídeo creado en Youtube por The New York times: The New York times Youtube edition, en el que en poco más de dos años se han realizado más de 365.000 reproducciones de sus vídeos. La tabla ocho muestra la presencia de los diferentes diarios en tres de estas plataformas.

http://www.youtube.com/user/TheNewYorkTimes

\section{Conclusiones}

La consolidación de la Web como una herramienta de trabajo, comunicación y entretenimiento a escala
2.0 y para llevar a cabo el proceso de selección se han tenido en cuenta dos aspectos:

- Sitios web que disfrutan de mayor popularidad entre los usuarios.

- Sitios web que se emplean de forma más habitual por los medios de comunicación analizados.

En relación con las redes sociales se ha examinado si los diarios objeto de estudio están presentes en: Facebook, LinkedIn y MySpace. La tabla siete muestra su presencia y alcance en estas redes.

\begin{tabular}{|l|c|c|c|}
\hline \multicolumn{1}{|c|}{ Medio } & Facebook & LinkedIn & MySpace \\
\hline http://www.elpais.com/ & Sí & Sí & No \\
\hline http://www.elmundo.es/ & Sí & Sí & No \\
\hline http://www.elperiodico.com/ & Sí & No & No \\
\hline http://www.lavanguardia.es/ & Sí & Sí & No \\
\hline http://www.abc.es/ & Sí & Sí & No \\
\hline http://www.lemonde.fr/ & Sí & Sí & No \\
\hline http://www.guardian.co.uk/ & Sí & Sí & No \\
\hline http://www.nytimes.com/ & Sí & Sí & Sí \\
\hline
\end{tabular}

Tabla 7. Presencia de los diarios digitales en redes sociales (análisis 20/11/2009) 


\begin{tabular}{|l|c|c|c|}
\hline \multicolumn{1}{|c|}{ Medio } & Youtube & Flickr & Twitter \\
\hline http://www.elpais.com/ & Sí & No & Sí \\
\hline http://www.elmundo.es/ & No & No & Sí \\
\hline http://www.elperiodico.com/ & Sí & No & Sí \\
\hline http://www.lavanguardia.es/ & Sí & No & Sí \\
\hline http://www.abc.es/ & No & No & Sí \\
\hline http://www.lemonde.fr/ & Sí & Sí & Sí \\
\hline http://www.guardian.co.uk/ & Sí & Sí & Sí \\
\hline http://www.nytimes.com/ & Sí & Sí & Sí \\
\hline
\end{tabular}

Tabla 8. Presencia de los diarios digitales en plataformas web 2.0 (análisis 25/11/2009)
- El empleo de nuevos lenguajes, por ejemplo contenidos multimedia.

- El aumento considerable de la información contextual a disposición del usuario mediante el uso del hipertexto.

- La participación directa de los usuarios en el proceso informativo a través de foros, encuestas y contribuciones que permiten ampliar o modificar una noticia o formular las preguntas de una entrevista mediante un encuentro digital.

Todo esto ha sido posible gracias a global, y la importancia que en ella cobran los contenidos de calidad han hecho de este medio un escenario ideal para la distribución de noticias.

Consciente de ello, la prensa digital ha ido aumentando progresivamente su presencia en la Web, hasta el punto de que hoy es difícil encontrar un diario de cierto prestigio que no posea una versión en línea.

No obstante, la mera presencia en la Web de estos medios no garantiza su éxito. La constante evolución de este entorno obliga a la prensa digital a adaptar sus contenidos y estrategias a las tecnologías y preferencias de los usuarios.

Con el fin de paliar este problema, en este trabajo se propone una lista de veintitrés indicadores que permiten evaluar si los sitios web de los diarios digitales utilizan los servicios y herramientas más adecuados para llevar a cabo su labor informativa.

El análisis llevado a cabo permite observar que en el proceso de adaptación al entorno digital los periódicos han prestado especial atención a la incorporación de herramientas de interacción, tales como la posibilidad de compartir y comentar las noticias, y al empleo de canales personalizados de difusión de la información (móvil, correo electrónico, etc.). Asimismo se constata una tendencia generalizada a la incorporación de los diarios en plataformas sociales como Facebook, Twitter, Flickr o Youtube.

\section{"La web social ha favorecido la evolución de los métodos y estrategias de comunicación utilizados hasta ahora por los medios"}

De este estudio se deducen además las siguientes tendencias: la consolidación de la web 2.0 o web social, que ha favorecido la evolución de los métodos y estrategias de comunicación utilizados hasta ahora por los medios. En este nuevo entorno el éxito de la prensa digital dependerá en gran medida de dos factores:

- Por un lado, su capacidad para crear sitios web que permitan la interacción tanto de los usuarios con los contenidos, como de los usuarios entre sí.

- Por otro, la web 2.0 pone a disposición de la prensa digital diferentes herramientas de comunicación social para llevar la noticia hasta sus potenciales usuarios. Su conocimiento y uso adecuado no sólo debería formar parte de la estrategia de los medios, sino que debiera ser uno de sus objetivos principales.

Por último, también será importante para los diarios digitales la adaptación de sus contenidos a nuevos formatos y plataformas, lo que les permitirá una mayor difusión de sus noticias y la captación de nuevas audiencias.

\section{Bibliografía}

Bradshaw, Paul. "How interactive are UK business news websites?". Online journalism blog, April 72008.

http://onlinejournalismblog.com/2008/04/07/how-interactive-are-uk-

Burke, John. "Is it possible to take AFP content off of Google News?". EditorsWeblog.org, July 202006.

http://www.editorsweblog.org/analysis/2006/07/is_it_possible_to_take_ afp_content_off_o.php

Codina, Lluís. "Metodología de análisis y evaluación de recursos digitales en línea".

http://www.lluiscodina.com/metodos.htm

Díaz-Noci, Javier. "Periodismo en internet: investigar los nuevos medios". En: López-García, Xosé; Pereira-Fariña, Xosé; Villanueva-Rey, Xosé (eds.). Investigar sobre periodismo: reunión científica de la Sociedad Española de Periodística (SEP), Santiago de Compostela, 27-28 de mayo de 2005. Santiago de Compostela: Universidade, Servizo de Publicacións e Intercambio Científico, 2005, pp. 74-100.

http://www.periodistica.es/imagenes/fotos/investigar_sobre_periodismo. $p d f$

Díaz-Noci, Javier et al. "Content and message analysis of online journalism: some methodological proposals". Trípodos, 2009, n. extra.

http://www.lluiscodina.com/onlineJournalism2009.pdf business-news-websites/ 
Díaz-Noci, Javier; Salaverría-Aliaga, Ramón. Manual de redacción ciberperiodística. Barcelona: Ariel, 2003.

Geary, Joanna. Interaction on business news websites (gráfico). 2008. http://www.joannageary.com/2008/04/06/interaction-on-business-newswebsites/

Glocer, Tom. "Old media must embrace the amateur". Financial times, March 82006.

http://www.ft.com/cms/s/0/2fd18348-ae49-11da-8ffb-0000779e2340.html

Guallar, Javier; Abadal, Ernest. "Evaluación de hemerotecas de prensa digital: indicadores y ejemplos de buenas prácticas". El profesional de la información, 2009, mayo-junio, v. 18, n. 3, pp. 255-269.

http://eprints.rclis.org/16899/

Guallar, Javier. "La renovación de los diarios digitales: rediseños y web 2.0". El profesional de la información, 2007, mayo-junio, v. 16, n. 3, pp. 235-242.

http://eprints.rclis.org/archive/00011822/01/epi07_guallar_renovaciondiarios-digitales.pdf

Horrigan, John B. For many home broadband users, the internet is a primary news source. Washington, DC: Pew Internet and American Life Project, 2006

Johnson, Bobbie. "Google News under fire". The guardian, March 2 2006.

http://www.guardian.co.uk/media/2006/mar/02/digitalmedia.news

Nielsen, Jakob. "Search engines as leeches on the Web". Useit.com, Alertbox, January 92006.

http://www.useit.com/alertbox/search_engines.html

López-García, Guillermo (ed.). Comunicación local y nuevos formatos periodísticos en internet: cibermedios, confidenciales y weblogs. Valencia: Servei de Publicacions de la Universitat de València, 2008. http://www.cibermediosvalencianos.es/ComunicacionLocal.pdf

Martínez-Rubio, Rosa. "La recuperación de la información en los periódicos digitales valencianos”. En: López-García, Guillermo. Comunicación local y nuevos formatos periodísticos en internet: cibermedios, confidenciales $y$ weblogs. Valencia: Servei de Publicacions de la Universitat de València, 2008, pp. 99-140.

http://www.cibermediosvalencianos.es/comloc/Martinez.pdf

Meso-Ayerdi, Koldo; Palomo-Torres, Bella. "Los blogs de autor en los ciberdarios de referencia españoles". Prisma.com, 2008, diciembre, n. 7. http://prisma.cetac.up.pt/edicao_n7_dezembro_de_2008/los_blogs_de_ autor_en_los_cibe.html

Palacios, Marcos. "La memoria como criterio de valoración de calidad en el ciberperiodismo: algunas consideraciones". El profesional de la información, 2009, mayo-junio, v. 18, n. 3, pp. 270-276.

Palacios, Marcos; Díaz-Noci, Javier (eds.). Online journalism: research methods: a multidisciplinary approach in comparative perspective. Bilbao: Servicio Editorial de la Universidad del País Vasco, 2009. ISBN 978-849860-191-6.

http://upf.academia.edu/JavierD\%C3\%ADazNoci/Books/88821/OnlineJournalism--Research-Methods
Pew Research Center's Project for Excellence in Journalism. The state of the news media 2007: Digital journalism: a topography of news websites by the project for excellence in journalism

http://www.stateofthenewsmedia.com/2007/narrative_digital_intro. asp? cat $=1$ \& media $=2$

Pew Research Center's Project for Excellence in Journalism. The state of the news media 2008: online.

http://www.stateofthenewsmedia.org/2008/narrative_online_intro. php?cat $=0 \&$ media $=5$

Pew Research Center's Project for Excellence in Journalism. The state of the news media 2009.

http://www.stateofthemedia.org/2009/index.htm

Rodríguez-Martínez, Ruth; Pedraza-Jiménez, Rafael. "Prensa digital y web 2.0". Hipertext.net, 2009, n. 7

http://www.hipertext.net/web/pag297.htm

Salaverría-Aliaga, Ramón; Díaz-Noci, Javier; López, Xosé; Palomo-Torres, Bella. "Spain: multimedia richness and variety of business models". En: Van der Wurff, Richard; Lauf, Edmund; O'Sullivan, John (eds.). Print and online newspapers in Europe: a comparative content analysis in 16 countries in Western and Eastern Europe. Amsterdam: Het Spinhuis, 2005, pp. 231-244.

Schutz, Tanjev. "Interactive options in online journalism: a content analysis of 100 US newspapers". Journal of computer mediated communication, 1999, v. 5, n. 1 .

http://jcmc.indiana.edu/vol5/issue1/schultz.html

Tancer, Bill. "Is Facebook the future of search?". Time magazine, Feb. 06 2008

http://www.time.com/time/business/article/0, 8599,1710493,00. html?xid=rss-business

The Bivings Group. The use of the internet by America's largest newspapers (2008 ed.).

http://www.bivingsreport.com/2008/the-use-of-the-internet-by-americaslargest-newspapers-2008-edition/

Voces-Merayo, Ramón. “¿Por qué la Web debe ser accesible?”. Hipertext. net, 2007, n. 5.

http://www.hipertext.net/web/pag278.htm

Wurff, Richard Van der; Lauf, Edmund; O'Sullivan, John (eds). Print and online newspapers in Europe: a comparative content analysis in 16 countries in Western and Eastern Europe. Amsterdam: Het Spinhuis, 2005.

Zamith, Fernando. Ciberjornalismo: as potencialidades da internet nos sites noticiosos portugueses. Porto: Afrontamento, 2008.

Ruth Rodríguez-Martínez, Lluís Codina, Rafael Pedraza-Jiménez. Universitat Pompeu Fabra.

ruth.rodriguez@upf.edu

lluis.codina@upf.edu

rafael.pedraza@upf.edu

\section{Suscripciones}

Renovar (o comenzar) la suscripción a "El profesional de la información" es ágil y sencillo.

Usted puede gestionar online su suscripción conectándose a esta web: http://www.elprofesionaldelainformacion.com/suscripciones.html

Si lo desea puede comunicar con nosotros dirigiéndose a: suscripciones@elprofesionaldelainformacion.com

o al teléfono: +34-609352954 